\title{
Method for Effective Use of Cloudlet Network Resources
}

\author{
Rashid G. Alakbarov \\ Institute of Information Technology of ANAS, Baku, Azerbaijan \\ E-mail: t.direktor_muavini@iit.science.az
}

Received: 07 October 2019; Accepted: 24 June 2020; Published: 08 October 2020

\begin{abstract}
The article addresses the issue of balanced placement of mobile software applications of mobile users in cloudlets deployed near base stations of Wireless Metropolitan Area Networks (WMAN), taking into account their technical capabilities. It is noted that the proposed model is more efficient in meeting the demand for computing and memory resources of mobile devices, eliminating network delays and using a reliable communication channel. At the same time, a minimum of cloudlet-based communication channels with a mobile user was suggested, reducing the network load and reliability of the communication channel when using multimedia software on mobile devices. The article reviews the balanced distribution of the tasks in the cloudlet network. If a user offloads the task to the nearest cloud and resolves it there, then the delays and energy consumption will be less. When the cloudlet is far from the mobile device, as the number of communication channels increases the delays are observed. Moreover, the article discusses the issue of selecting the cloudlets that meet some of the user requirements. Using the possible values that determine the importance of cloudlets (vacant resources in cloudlets, closeness of cloudlets to the user, high reliability, etc.), the conditions, according to which the user's application is offloaded to the certain cloudlet, are studied and a method is proposed.
\end{abstract}

Index Terms: Mobile cloud computing, mobile devices, cloudlet, communication channel, multimedia software applications, wireless communication channel, energy consumption.

\section{Introduction}

Effective use of cloudlets on the second level of hierarchically structured mobile computing clouds has been analyzed. At the same time, issues of meeting the demand for computing and memory resources of mobile devices using this technology were researched. One solution to the problem of efficient use of cloudlet resources located near base stations of wireless metropolitan area networks is given. It is established that the number of communication channels between the user and the cloudlet must be minimal for the efficient use of cloudlet resources in mobile computing clouds. The conditions for selecting cloudlets for downloading necessary software applications were researched and the method was proposed using the possible values that determine the importance of the cloudlets (the user's proximity to the cloud, available resources, etc.).

Although centralized (traditional) clouds currently used by the users have high computing and memory resources, they are not able to rapidly transmit processed data to users. Mobile users connect to Cloud Computing system using base stations through Cellular Networks (3G/4G) and Wi-Fi (Access Point) and use its computing and memory resources. The rapid increase of a number of mobile users in computing clouds creates the possibility of network overload, which causes great delays in delivering processed data to the user. In order to eliminate noted shortcoming, it is necessary to locate computing clouds' resources near the user. Cloudlets are used on the 2nd level of the hierarchically structured network. Cloudlets located near to the users provide faster processing of user data in comparison with further located cloud servers. This type of connection provides less loading of the network and faster acquisition of processed data [1]. Determining which base station to locate the cloudlets near to and what characteristics they should attain and determining the connection of the user to the closer cloudlet are among urgent issues. Thus, cloudlets will be created near base stations of mobile operators to make greater use of mobile technology in cloud technologies. Cloudlet (small computing clouds) is a device (server) that is located close to the user, enabling the user to process applications faster. Cloud services that users need are implemented through cloudlets, which improves the quality of services [2]. At the same time, ensuring the minimum communication channels between the cloud and the user used is a topical issue. The smaller number of channels between the user and the cloudlet increases the reliability and channel bandwidth and reduces the power consumption of the mobile device.

Numerous cloudlets of the cloudlet-based Wireless Metropolitan Area Networks (WMAN) are used. Cloudlet- 
based network RMC contains information about the technical capacity of computer (server, desktop, notebook, etc.). Moreover, RMC collects information about the technical capacity of cloudlets (processor frequency, number of processor cores, number of virtual machines and their technical characteristics, storage capacity, etc.) and which base station the cloudlets are close to within the mobile network. Therefore, according to the received query, it is important to know in which cloudlet or virtual machine the user can place the application called from the cloud servers that meets the user's requirements. In many cases, users do not choose the type of virtual machine according to the resources required to solve the task. By providing the users with the solution of queries in nearby cloudlets, it is possible to eliminate the delays by reducing the number of communication channels between users and cloudlets and ensure reliable operation of the network. Proper deployment of software applications used by users in the cloud may reduce the energy consumption, latency and interruptions.

\section{Summary of Related Works}

In recent years, there has been intensive research work on the application of various network technologies for mobile users, especially the advantages of networks created by the application of cloud technologies, and solution of the problems that arise in these networks.

Article [3] is dedicated to the effective solution of set issues by storing software and relevant data on cloud servers using cloud technology. Article [4] is dedicated to problems of bringing cloud computing closer to mobile devices by applying new technologies such as Mobile Edge Computing (MEC). In this case, applying selective ABSO strategy applied based on ARIMA-BP allows minimizing energy consumption by mobile devices. The article also provides the results of the stimulation process in order to demonstrate the advantages of the indicated method. In many studies, it is of interest to determine the location and timing of cloud users' creation of cloudlets, as well as how to effectively use cloudlet resources. CPU usage percentage, memory usage and performance time of main operations are analyzed. Noting the significance of the selected parameters, the algorithm for calculating integral monitoring parameters is provided. In accordance with monitoring results, a method for determining time and place of cloudlets near the base station is provided [5, 6]. Some studies [7] research issues such as planning of geographically distributed network, optimal placement of cloudlets near base stations and optimization of network parameters. Some studies provide a solution of issues that determine the response time of loading issues and optimal distribution issue of problems among cloudlets and minimize response period on cloudlets. Authors provided a scaleable, rapid algorithm for problem solution [8]. Article [9] discusses the solution of issues such as joint usage of cloud resources based on cloud computing and creating placement algorithms. Many experiments are conducted based on Cloud Sim simulator. Article [10] demonstrates that loading problems on the most suitable cloudlet are among key issues. The article discusses selecting a cloudlet that is relevant to the problems to be solved in multicloudlet environment. Here, a cloudlet that matches the type of problem to be solved in selected among multiple cloudlets in the proximity of the user. It is noted that the proposed strategy can result in reduced energy consumption in mobile devices. Users problems can be solved by providing a nearby cloudlet, eliminating delays by reducing communication channels among users and cloudlets and providing reliable operation of the network [11, 12]. It is possible to reduce energy consumption, delays and disconnections by correctly placing interface and main parts of the software used by the users relevantly on the mobile devices and cloud servers [13]. Some researchers note long-term delays in data exchange among the user and remote cloud due to physical distance between the cloud server and the users [14]. Close placement of cloud servers to the users significantly reduces delays in data exchange [15]. Some researchers proposed that a small number of communication channels among user and cloudlet provide the performance of software with high quality, minimal delays and minimal disconnections [16].

Article [17] has set a solution for the problem of selecting virtual machines that can provide a faster solution to problems in accordance with user requirements using technical capabilities of cloudlets and virtual machines created in cloudlets. Article [18] also explores the optimal distribution of mobile software applications among the mobile network's cloud servers. It has been studied that the bandwidth of communication channels has a significant impact on the speed of mobile applications operation [19]. Some studies [20, 21] have focused on the creation of cloudlet network infrastructure near the access points of wireless metropolitan area networks. Some studies [22] examined the conditions that necessitate the creation of cloudlets in mobile computing clouds and looked into forecasting which base stations the cloudlets would be located in. Article [23] addresses the problem of reducing energy consumption by the optimal distribution of a user-solved problem between a cloud server and a remote cloud server. Some researchers have looked at the problem-solving process by distributing software applications across multiple cloudlets [24]. Some authors have addressed the issue of migrating services on mobile clouds (deploying services in clouds closer to users), and have shown some advantages of this method. In the event of a need for higher priority software storage, a stored but relatively little used software tool can be removed from the cloud resources [25]. The article [26] addresses the creation of computational resources (virtual machines) using hierarchical analysis techniques for the efficient use of computing cloud environments. Article [27] proposes the use of cloudlet-based cloud computing to eliminate the delays caused by overload in mobile cloud computing and a method of efficient use of cloudlets. It is [28] suggested to use cloudlets to troubleshoot problems with mobile device batteries and computing resources, and to eliminate delays in resolving user 
software applications in cloudlets. The article [29] discusses the structural problem of a cloudlet-based network. The article suggests how to create a cloudlet network in Wireless Metropolitan Area Networks in order to solve the problem of where and how many cloudlets should be deployed.

\section{Creation of Hierarchically Structured Network Infrastructure on the Basis of Cloudlets}

The establishment of cloudlet network infrastructure near the Wireless Metropolitan Area Networks (WMAN) is a topical issue. It is best to create cloudlets near all access points of the specified network. It is not economically viable and increases network cost. On the other hand, there may be little or no use of cloudlets placed near certain access points. Therefore, cloudlets are placed near the locations where most mobile users are (shopping centers, libraries, schools, universities, stadiums, stations, airports, etc.).

Cloudlets are created based on computers with different technical characteristics (server, workstation, desktop computer, netbook, etc.). If the cloud computing and storage resources near any Access Point are not able to handle the inquiries of a large number of mobile users, then the mobile user will have to use the resources of other nearby cloudlets. At the same time, cloud resources located near a mobile user may not have available resources while being used by other users. In this case, the mobile user needs to use the required resources of other cloudlets that are close to them. Studies have shown that centralized mobile Cloud Computing systems do not provide quality services to users. That is, in this type of mobile computing systems, situations such as network overload, delayed delivery to the user, expensive service, delays, traffic overload, etc. do not allow obtaining quality services. Hierarchical (3-level) mobile Cloud Computing systems are used to address these problems [30, 31]. In the proposed architecture, mobile internet users download and use the application software they need to their nearby cloudlet systems' servers, which in its turn frees the Internet network from overloading. This architecture, although partly, improves the performance of some of the specified parameters. In the hierarchically structured architecture, Cloud Computing servers are deployed at the 1st level, cloudlets near base stations are used on 2nd level and mobile users are placed on 3rd level.

Advantages of using cloudlets include faster access to

the service, mobility support, and reduced roaming costs. Because user's software applications are located in nearby cloudlets, users can gain access to information immediately, eliminating network communication delays. Also, as in cloud computing, the technical capabilities of cloudlets are higher than the technical capabilities of mobile devices, which enable the operation of applications that cannot be processed on mobile devices. If the resources of a cloudlet located near the cloud user do not allow the problem to be solved, then another cloudlet will be selected to resolve the issue.

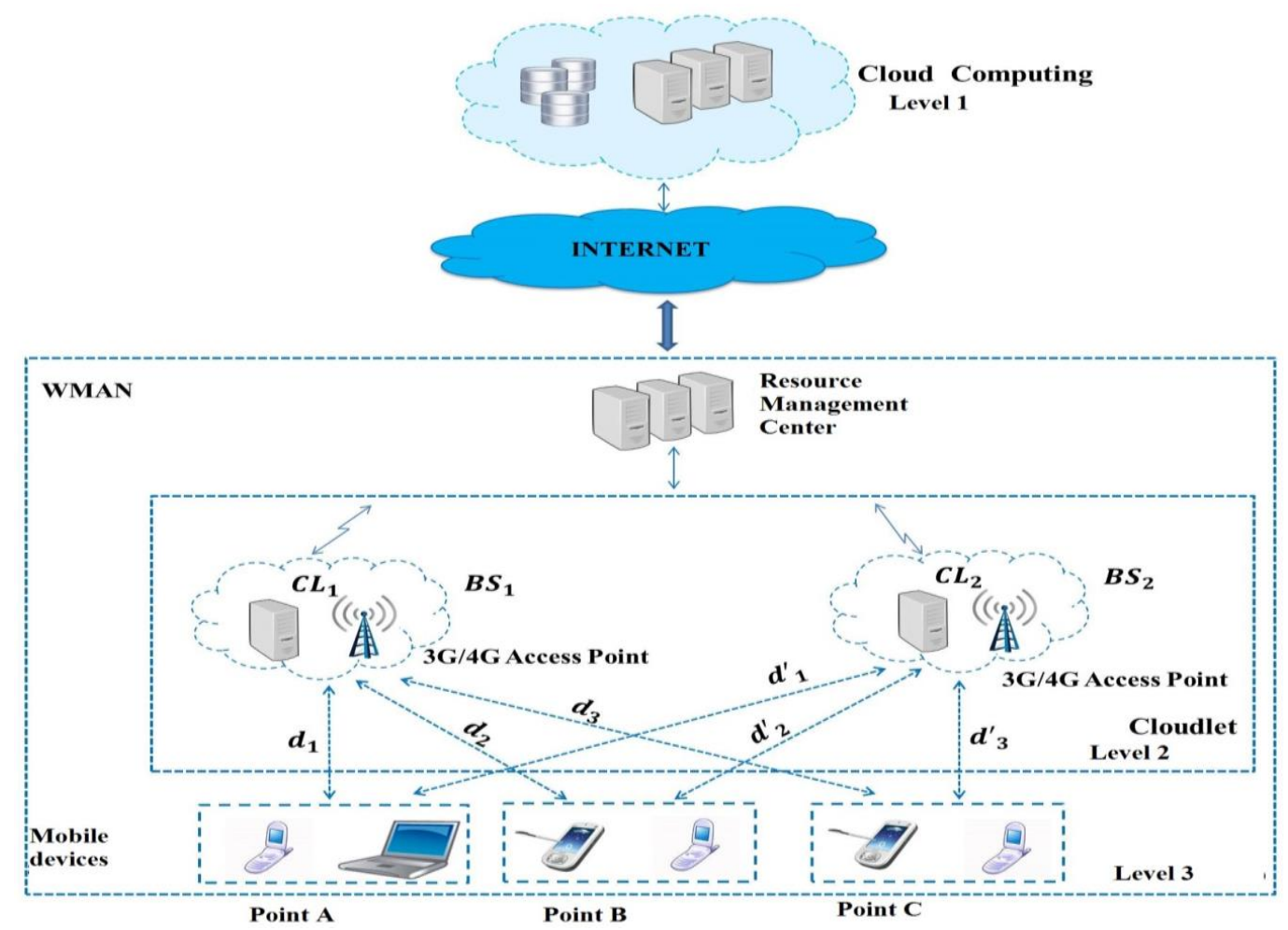

Fig.1. Cloudlet-based hierarchically structured mobile cloud computing 
One way to effectively use cloudlet resources is to download and uses frequently used software applications (games, translation programs, etc.) in the cloudlet, and keep them there for as long as this application remains relevant. It should be noted that the placement of mobile users' software applications in a cloudlet that is close and will remain connected to it for a long time allows for faster and more efficient performance. Proximity is determined by the number of cloudlet-user communication channels. Qualitative service of cloudlets near base stations depends on the technical capabilities of computer hardware used in its creation (processor speed, number of cores and virtual machines, memory capacity, network bandwidth, etc.). This is because the technical capabilities of cloudlets should allow the user to run the software application that they use. A cloudlet created using a Resource Management Center (RMC) to address the identified shortcomings was mainly offered by a hierarchical mobile computing cloud (Figure 1).

Resource Management Center (RMC) of a hierarchical network that provides information on the location and technical capabilities of cloudlets. Cloud services that users need are implemented through cloudlets, which improves the quality of their services. When mobile users sign in to the cloudlet network, they first connect to the RMC, which then connects the user to the relevant remote servers for a short period of time. Mobile devices connect to the cloudlet network via $3 \mathrm{G} / 4 \mathrm{G}$ or Wi-Fi. Inquiries and information from mobile users (e.g. ID and location) are transmitted to RMC that provides mobile network services. Subsequently, inquiries from subscribers are sent to the server of the proposed model located in the RMC that provides Internet connection and a connection is established with the relevant cloud over the Internet with its assistance. The Management Center contains information about computer equipment (server, desktop, notebook, etc.) used to create cloudlets. At the same time, the management center collects information about the technical capabilities of cloudlets (processor clock speed, the number of processor cores, the number of virtual machines and their specifications, the amount of memory, etc.) and which cloudlet the users are located nearby to on the mobile network. Therefore, in accordance with the incoming inquiry, the software application that is called from the cloud servers will be deployed on cloudlets that meet the user's requirements. As can be seen from the diagram, mobile internet users download and use the applications they need on the cloudlet systems servers that are close to them, which in turn frees the Internet from overloading. This architecture, in part, improves the performance of some of the specified parameters (problem resolution time, communication channel delays, disconnections, power consumption, etc.). In the hierarchical structure, servers of the computing cloud system are on level 1 and cloudlets placed near base stations are on level 2. Thus, a cloudlet is created near base stations of mobile operators to make greater use of mobile equipment in cloud technologies.

\section{Problem Statement}

The efficient use of cloudlets located near the base stations of Wireless Metropolitan Area Networks (WMAN) was considered. The reliability and technical capabilities of the equipment used in the creation of cloudlets vary. The user requires to solve the task in the cloud with high reliability. On the other hand, the user requires to connect to the cloudlet of his/her choice with a minimum number of communication channels. The small number of communication channels allows for less interruptions and better security. Placing cloudlets near all base stations of WMAN is not economically feasible and increases the cost of the network. Therefore, by monitoring, the cloudlets are placed near the locations where there are more mobile users (airports, stadiums, universities, shopping malls, etc.). on the other hand, there may not be vacant virtual machine in the cloudlet near the user that satisfies his/her request. In this case, the user uses more remote cloudlet. In this case, communication channels between the remote cloudlet and the user must be minimal. Because a large number of communication channels leads to the network overload and latency. The article discusses the issue of selecting the cloudlet in accordance with the conditions set by the user. According to the user's request, RMC identifies the vacant cloudlets in the cloudlet network, then selects a set of cloudlets that match the user's reliability requirements. The cloudlet closest to the user (minimum link channel) is selected out of the selected cloudlet and the task is sent to that cloudlet to be solved.

Let's review the connection of the mobile user to the cloudlet in cloudlet network. The article provides a solution for the selection of a cloudlet considering some parameters (proximity, reliability, available resources, minimum communication channels etc.). Coordinates of the cloudlets located in a wireless metropolitan area network can be written as follows:

$$
\begin{aligned}
& C_{1}\left(X_{1}, Y_{1}\right) \text {-first cloudlet coordinates; } \\
& C_{2}\left(X_{2}, Y_{2}\right) \text { - second cloudlet coordinates; }
\end{aligned}
$$

Mobile user can write down the coordinates of the points as follows:

$$
\begin{aligned}
& A\left(X_{a}, Y_{a}\right) \text {-coordinates of point } A ; \\
& B\left(X_{b}, Y_{b}\right) \text { - coordinates of point } B ; \\
& C\left(X_{c}, Y_{c}\right) \text { - coordinates of point } C ;
\end{aligned}
$$


Let's mark the maximum connection distance between a base station in the mobile network and mobile device as $R$ (mobile devices signal receiving range). Let's mark the existing distances between mobile users in points $A, B$ and $C$, and base stations ( $B S_{1}$ and $B S_{2}$ ) as $d_{1}, d_{2}, d_{3}$ and $d_{1}^{\prime}, d_{2}^{\prime}, d_{3}^{\prime}$ correspondingly. If noted existing distances are less than $R$, then mobile devices are connected to the base station. Considering distances $d_{1}$ and $d_{2}$ are less than $R$ ( $B S_{1}$ coverage area), problems of users in points $A$ and $B$ can be solved by uploading them to cloudlet $C_{1}$. On the other hand, mobile users in point $B$ have an option to connect to cloudlet $C_{2}, d_{2}^{\prime}<R$. But as point $B$ is closer to $B S_{1}$, $d_{2}<d_{2}^{\prime}$, users of point $B$ are initially connected to $C_{1}$. Distance between mobile users and cloudlet is defined as Euclidian distance. Distance between mobile users in points $A, B$ and $C$ and cloudlet $C_{1}, d_{1}, d_{2}, d_{3}$, is determined as following:

$$
\begin{aligned}
& d_{1}=\left\|A\left(X_{a}, Y_{a}\right)-C_{1}\left(X_{1}, Y_{1}\right)\right\|=\sqrt{\left(X_{a}-X_{1}\right)^{2}+\left(Y_{a}-Y_{1}\right)^{2}} \\
& d_{2}=\left\|B\left(X_{b}, Y_{b}\right)-C_{1}\left(X_{1}, Y_{1}\right)\right\|=\sqrt{\left(X_{b}-X_{1}\right)^{2}+\left(Y_{b}-Y_{1}\right)^{2}} \\
& d_{3}=\left\|C\left(X_{c}, Y_{c}\right)-C_{1}\left(X_{1}, Y_{1}\right)\right\|=\sqrt{\left(X_{c}-X_{1}\right)^{2}+\left(Y_{c}-Y_{1}\right)^{2}}
\end{aligned}
$$

Analogically, $d_{1}^{\prime}, d_{2}^{\prime}, d_{3}^{\prime}$ distance between mobile users in points $A, B$ and $C$ and cloudlet $C_{2}$ can be determined using as shown above.

Users in point A can connect to $B S_{1}$ the base station, as $d_{1}<R$ and cannot connect to the station $B S_{2}$, as $d_{1}^{\prime}>R$. Mobile users in point $C$ can connect to the base station $B S_{2},\left(d_{3}^{\prime}<R\right)$ and cannot connect to the station $B S_{1}\left(d_{3}>R\right)$. Users in point B can connect to both $B S_{1}$ and $B S_{2}, d_{2}<d_{2}^{\prime}<R$. But, due to $d_{2}<d_{2}^{\prime}$, they are initially connected to $C L_{1}$ cloudlet.

A small number of communication channels between the user and the cloudlet can lead to faster processing of the problem and less energy consumption on the mobile device. Given the above, the article prefers to select a cloudlet with one communication channel between the user and the cloudlet.

The mobile device is connected to the close base station by meeting $d<R$ condition on wireless metropolitan area network, here $R$ - is the maximum connection range (distance) of the mobile device to the base station, $d-$ is the existing distance between the mobile user and the base station.

$K$ number of mobile users can connect to the base station during the $T$ period.

Due to scarce cloudlet resources connected to base stations, it can server $N<K$ number of mobile users.

$N$ - is the current number of users served by the cloudlet. For example: if 100 virtual machines are created on the cloudlet connected to the base station, then it can only process 100 mobile user problems in a certain $t$ moment.

The software application of the 101th connected mobile user cannot be downloaded and solved at noted cloudlet. Only after one of $100 \mathrm{VMs}$ is freed, the problem of 101st user can be solved at that cloudlet. But in some cases, other base stations can be near the mobile user (in our case, the user of point $\mathrm{B}$ ) that meets $d_{1}<d_{2}^{\prime}<R$ condition. Namely, there is a set of mobile users connected to the 1st base station, which can connect to 2nd base station within the connection range $(R)$ and use the resources of the cloudlet connected to it. In this case, Resource Management Center connects those mobile users with the 2 nd base station and loads their software applications to the 2nd cloudlet and obtains available resources on the 1st cloudlet and solves the problems of the next (101st) user by connecting to it using one communication channel. In this case, the number of connection between the mobile user and the cloud is at minimum (one communication channel).

Let's present possible mobility scenarios for the mobile user in the cloudlet network. Connection distance of mobile users in point $A$ to $B S_{1}$ base stations are $d_{1}<R$. Mobile users in point A cannot connect to $\left(B S_{2}\right),\left(d_{1}^{\prime}>R\right)$. Mobile users in point $C$ cannot connect to $B S_{1},\left(d_{3}>R\right)$. As seen from the diagram, mobile users at point $B$ can connect to $B S_{1}$ and $B S_{2}$. Namely, $d_{1}<d_{2}^{\prime}<R$. As $d_{1}$ connection distance of mobile users at point $\mathrm{B}$ is smaller, they are initially connected to the base station $B S_{1}$ and start using the resources of cloudlet $C L_{1}$. After the passage of a certain $T$ period of time, as the number of users increases (in point A and B), resources of $C L_{1}$ a cloudlet is not sufficient to serve. In this case, the Resource Management Center directs the users of point $B$ connected to $B S_{2}$ base station to cloudlet $C L_{2}$ and implements problem solution there.

Thus, at point $A$, new users join $C L_{1}$ through a single communication channel, while users at point B also connect to the $C L_{2}$ cloudlet through a single communication channel. This, in turn, ensures that the user and the cloudlet use a 
quality communication channel. When using one channel, delays are eliminated and the proximity of software applications to the mobile user reduces energy consumption. If users do not have a single communication channel in the vicinity, then mobile users connect to remote cloudlets using multiple communication channels. This leads to an increase in energy consumption, causing disconnections and delays.

Thus, the proposed method in part, minimizes delays in communication channels, eliminates the limitations of computing and memory resources of mobile devices, speeds up the solution, and reduces the power consumption of mobile devices. In the proposed model, mobile internet users download and use the application software they need to close clouds, which in turn frees the Internet network from overloading. On the other hand, in hierarchically structured mobile computing clouds, the security of communication channels is more reliable, as the software is located on cloudlets closer to the mobile user. Because in the centralized mobile computing clouds, the connection between the mobile user and the remote cloud server is over the Internet, the intensity of unauthorized access to the communication channels is increasing, and providing security is difficult.

\section{Problem Solution}

Numerous cloudlets of the cloudlet-based Wireless Metropolitan Area Networks (WMAN) are used. Cloudletbased network RMC contains information about the technical capacity of computer (server, desktop, notebook, etc.). Moreover, RMC collects information about the technical capacity of cloudlets (processor frequency, number of processor cores, number of virtual machines and their technical characteristics, storage capacity, etc.) and which base station the cloudlets are close to within the mobile network. Therefore, according to the received query, it is important to know in which cloudlet or virtual machine the user can place the application called from the cloud servers that meets the user's requirements. In many cases, users do not choose the type of virtual machine according to the resources required to solve the task. By providing the users with the solution of queries in nearby cloudlets, it is possible to eliminate the delays by reducing the number of communication channels between users and cloudlets and ensure reliable operation of the network. Proper deployment of software applications used by users in the cloud may reduce the energy consumption, latency and interruptions. The time spent on the task solution and delivery to the user depends on the computing power of the virtual machine created in the cloudlets and the number of nodes between the cloudlet and the user. The smaller the number of nodes between the cloud and the user, the less the delays are. This, in turn, helps deliver the results and information to the user faster. Before selecting the cloud, you need to inspect the network and determine the status. Thus, after the inspection, the information about the loading and technical capabilities of cloudlets is obtained, and when the request is received, it is determined in which cloudlet the task will be resolved faster. The number of communication channels between users and the cloud should be minimal, which ensures reliable operation of communication channels. Moreover, the small number of communication channels reduces delays and increases the reliability of security issues.

Assume that in local cloudlet system $N$ number of cloudlets are connected Resource Management Center (RMC). Geographic location coordinates, the computing power of these cloudlets and reliability of each individual cloudlet are known to RMC. Cloudlet location coordinates are

$$
C_{1}\left(x_{1}, y_{1}\right), C_{2}\left(x_{2}, y_{2}\right), \ldots, C_{N}\left(x_{N}, y_{N}\right)
$$

Creating the following set:

$$
C=\left\{C_{n}\left(x_{n}, y_{n}\right)\right\}, n \in[1, N]
$$

For simplicity, the computing power is the speed (productivity) of the processor, reliability indicator is experimental reliability of the cloudlet and communication facilities.

When users connect to cloudlets, they must be provided with available computing power to resolve a user's problem in the selected cloudlet. The computing power of cloudlets is determined by the virtual machines created there. Since each user is associated with a virtual machine, the presence of an available virtual machine simply means the presence of available computing power in the cloudlet. Therefore, we can mark the number of available virtual machines in $\mathrm{n}^{\text {th }}$ cloudlet with $v_{n}$. Here we assume that the characteristics of these machines are sufficient to solve the problems of users.

Reliability of communication devices of each cloudlet can be calculated in RMC using a simple method. Let's mark the total number of inquiries made on $\mathrm{N}^{\text {th }}$ cloudlet during exploitation period with $m_{n}$, and the number of unsuccessful inquiries made during this period with $\varepsilon_{n}$. After each inquiry

$$
m_{n}=m_{n}+1
$$


After each effective result

$$
\varepsilon_{n}=\varepsilon_{n}+1
$$

Then reliability of the $\mathrm{n}^{\text {th }}$ cloudlet can be calculated as

$$
p_{n}=1-\frac{\varepsilon_{n}}{m_{n}}
$$

This simple indicator can be taken as a measure of the reliability of the cloud, although it is not an accurate index of the cloudlet's reliability. The main advantage of this index is that it can be easily calculated for each cloudlet in RMC. The following can be noted about this reliability index of Cloudlets:

1. The specified algorithm can only be implemented for inquiries incoming from users connected to the cloudlet. The algorithm is not implemented for inquiries incoming from users who are not connected or want to connect to the cloudlet.

2. This reliability indicator is an integrated reliability indicator for the applied cloudlet. Failure reasons for inquiries are irrelevant. Failure can be caused both by the cloudlet itself and by communication channels or otherwise.

3. It is known that RMC connects mobile users to one of the virtual machines inside the cloudlet. Therefore, the reliability index can be calculated for each virtual machine (cloudlet).

A large number of virtual machines can be built in each cloudlet used in the network within its technical capabilities. Thus, $C=\left\{C_{n}\left(x_{n}, y_{n}\right)\right\}, n \in[1, N]$ cloudlet set can be determined using $V=\left\{v_{n}\right\}$ available virtual machines set and $P=\left\{p_{n}\right\}$ reliability indicators' set. Cloudlet set can also be denoted as

$$
C=\left\{C_{n}\left(x_{n}, y_{n}\right),\left\{v_{n}\right\},\left\{p_{n}\right\}, n \in[1, N]\right\}
$$

with its characteristics. Here it must be noted that depending on the solution of certain problems, other characteristics of cloudlets can be engaged in the analysis process. Besides, new characteristics can be determined and calculated in other necessary cases.

Assume that where $\forall j \in[1, J], J$ number $U_{i}$ mobile stationary users are connected to the cloudlet network determined by RMC in (2) at the current moment. These users form

$$
U=\left\{U_{j}\left(x_{j}, y_{j}\right)\right\}
$$

set with their coordinates. As in cloudlet set, user set also needs (requires) computing resources and reliable indicators of the cloudlet each user will be connected to. If we mark these needs as $v_{i}, p_{i}$ for $\mathrm{J}^{\text {th }}$ user, then user set must be determined as

$$
U=\left\{U_{j}\left(x_{j}, y_{j}\right),\left\{v_{j}\right\},\left\{p_{j}\right\}, j \in[1, J]\right\}
$$

In addition to abovementioned, each user can connect to cloudlets no farther than $d$ (connection distance of the mobile user to base station), with no more than $k_{j}$ communication channels (for example 2 communication channels) in connection. When these parameters are added, the user settings can be determined as

$$
U=\left\{U_{j}\left(x_{j}, y_{j}\right),\left\{v_{j}\right\},\left\{p_{j}\right\}\{d\},\left\{k_{j}\right\}, j \in[1, J]\right\}
$$

There can be several consequently connected communication channels among the user and selected virtual machine or cloudlet. A multitude of communication channels reduce reliability indicators [32].

We can determine the distance between a $\mathrm{j}^{\text {th }}$ user and $\mathrm{n}^{\text {th }}$ cloudlet as Euclidian distance and mark it as $d_{j n}$

$$
d_{j n}=\sqrt{\left(x_{j}-x_{n}\right)^{2}+\left(y_{j}-y_{n}\right)^{2}}
$$


Considering abovementioned, the following conditions must be met with $k_{j n}$ a number of communication channels from $\mathrm{j}^{\text {th }}$ user to $\mathrm{n}^{\text {th }}$ cloudlet:

$$
\left\{\begin{array}{c}
d_{j n} \leq d, \\
k_{j n} \leq k_{j}, \\
v_{j} \leq v_{n}, p_{j} \leq p_{n}
\end{array}\right.
$$

The first line of (6) conditions belongs to distance parameter between user and cloudlet, second line to a number of communication channels between user and cloudlet, the third line to available virtual machines and reliability parameter

It must be noted that perhaps $\mathrm{j}^{\text {th }}$ user could connect to another cloudlet in case of availability of other cloudlets meeting (6) conditions and successfully implement the issues (functions). But RMC connects this user to the cloud with the most optimal connection. Below, we will use user characteristics to connect to other cloudlets.

Connection of new users to the set of cloudlets. Let's assume a new $\mathrm{J}+1^{\text {th }}$ user wants to connect to the set of cloudlet presented in (2). Coordinates of these users and parameters as determined in (3)-(4) are known to RMC. Let's mark coordinates $\left(x_{J+1}, y_{J+1}\right)$ and other necessary parameters of this user as $v_{J+1}, p_{J+1}, d, k_{J+1}$. Thus, new $U_{J+1}\left(x_{J+1}, y_{J+1}\right)$ is determined. $\mathrm{J}+1^{\text {th }}$ user can connect to a cloudlet that meets the following conditions:

$$
\left\{\begin{array}{c}
d_{J+1, n} \leq d, \\
k_{J+1, n} \leq k_{J+1}, \\
v_{J+1} \leq v_{n}, p_{J+1} \leq p_{n}
\end{array}\right.
$$

It is clear that there are certain sets of cloudlets that meet each presented condition. Let's mark the sets of cloudlets that meet the conditions in accordance with order number as following:

$$
\begin{gathered}
C_{1}=\left\{C_{n}\left(x_{n}, y_{n}\right), v_{J+1} \leq v_{n}, n \in[1, N]\right\} \\
C_{2}=\left\{C_{n}\left(x_{n}, y_{n}\right), p_{J+1} \leq p_{n}, n \in[1, N]\right\} \\
C_{3}=\left\{C_{n}\left(x_{n}, y_{n}\right), k_{J+1, n} \leq k_{J+1}, n \in[1, N]\right\} \\
C_{4}=\left\{C_{n}\left(x_{n}, y_{n}\right), d \leq d_{J+1}, n \in[1, N]\right\}
\end{gathered}
$$

For this reason, $\mathrm{j}+1^{\text {th }}$ user can connect to

$$
C_{J+1}=C_{1} \cap C_{2} \cap C_{3} \cap C_{4}
$$

set of cloudlets.

Where $C_{J+1}=\phi$, RMC cannot connect the new user to the provided set of cloudlets, as one or several of necessary (7) conditions are not met. In this case, there are many voids that match conditions that are not met in sets (8) - (11). For this reason, in order to solve this problem by freeing one or more users connected to cloudlets determined by $C_{3} \cap C_{4}$ set, RMC can connect them to other cloudlets that belong to a set that meets certain conditions (using one or two communication channels). In this case, $\mathrm{j}+1^{\text {th }}$ user can connect to $C_{J+1}$ set of cloudlets.

\section{Future Research}

Since mobile devices play an important role in people's lives, numerous research areas are available in the field of cloudlet-based mobile computing. Future research will predict which base stations to place the cloudlets close to in cloudlet-based mobile computing clouds; to identify and pre-deploy high-intensity software applications in cloudlets; to optimally place mobile applications of the moving mobile user in cloudlets in accordance with the routing; and to use cloudlets efficiently and in a balanced way; to deploy the user's application in the nearest cloudlet, etc. In the future studies, it is planned to develop the algorithms to solve abovementioned problems and experimentally test the results. 


\section{Conclusion}

The article provides a solution for mobile users' efficient use of cloudlets resources in wireless metropolitan area mobile computing clouds. At this time, the capabilities of most of the cloudlets on the network were analyzed together with some of the characteristics.

The article discussed the issue of selecting cloudlets according to some user requirements. Using the connection distance from the mobile device to the cloudlet (mobile device's signal reception range) and the possible values that determine the importance of cloudlets (user proximity, high reliability, etc.), the conditions for offloading the user's application to certain cloudlet were studied. A cloudlet selection strategy in accordance with the user requirements was developed and a solution to the problem of balanced task distribution among the cloudlets was proposed. At the same time, mobile users are also listed along with their coordinates and other necessary features. A solution is provided for selection of a cloudlet that meets the user requirements among a multitude of cloudlets that meet different criteria. In order to provide a reliable solution to user problems, it is given preference to have a minimum number of communication channels with cloudlet. A method that ensures the connection of the mobile user to the cloudlet with a reliable communication channel is provided.

The presented method can be used to solve analogical problems.

\section{References}

[1] T. Diaby, B. B. Rad, "Cloud Computing: A review of the Concepts and Deployment Models," International Journal of Information Technology and Computer Science, vol. 9, no. 6, pp. 50-58, 2017.

[2] M. Goyal, S. Singh, "Mobile Cloud Computing," International Journal of Enhanced Research in Science Technology \& Engineering, vol. 3, no. 4, pp. 517-521, 2014.

[3] Y. Hao, M. Chen, L. Hu, M. S. Hossain, A. Ghoniem, "Energy Efficient Task Caching and Offloading for Mobile Edge Computing," IEEE Access, vol. 6, pp. 11365-11373. 2018.

[4] M. Zhao, K. Zhou, "Selective Offloading by Exploiting ARIMA-BP for Energy Optimization in Mobile Edge Computing Networks," Algorithms, vol. 12, no. 2, pp.1-13, 2019.

[5] H. T. Dinh, C. Lee, D. Niyato, P. Wang, "A survey of mobile cloud computing: Architecture, applications, and approaches," Wireless Communications and Mobile Computing, vol. 13, no. 18, pp. 1587-1611, 2013.

[6] R. Alakbarov, F. Pashayev, O. Alakbarov, "Forecasting Cloudlet Development on Mobile Computing Clouds" International Journal of Information Technology and Computer Science (IJITCS), vol. 9, no. 11, pp. 23-34, 2017.

[7] M. Mam, G. Leena, N. S. Saxena, "Improved K-means Clustering based Distribution Planning on a Geographical Network," International Journal of Intelligent Systems and Applications (IJISA), vol. 9, no. 4, pp. 69-75, 2017.

[8] M. Jia, W. Liang, Z. Xu, and M. Huang, "Cloudlet Load Balancing in Wireless Metropolitan Area Networks," IEEE INFOCOM 2016 - The 35th Annual IEEE International Conference on Computer Communications. p. 1-9, 2016.

[9] G. T. Hicham, E. A. Chaker, "Cloud Computing CPU Allocation and Scheduling Algorithms Using CloudSim Simulator," International Journal of Electrical and Computer Engineering, vol. 6, no. 4, pp. 1866-1879, 2016.

[10] D. G. Roy, D. De, A. Mukherjee, R. Buyya, "Application-aware cloudlet selection for computation offloading in multi-cloudlet environment," The Journal of Supercomputing, vol. 73, pp. 1672-1690, 2017.

[11] R. K. Alakbarov, F. Pashayev, M. Hashimov, "Development of the Method of Dynamic Distribution of Users' Data in Storage Devices in Cloud Technology," Advances in Information Sciences and Service Sciences, vol. 8, no. 1, pp. 16-21, 2016.

[12] O. P. Akomolafe, M. O. Abodunrin, "A Hybrid Cryptographic Model for Data Storage in Mobile Cloud Computing," I. J. Computer Network and Information Security, no. 6, pp. 53-60, 2017.

[13] Y. C. Shim, "Effects of cloudlets on interactive applications in mobile cloud computing environments," International Journal of Advanced Computer Technology, vol. 4, no.1, pp. 54-62, 2015.

[14] M. Satyanarayanan, P. Bahl, R. Caceres, N. Davies, "The case for vm-based cloudlets in mobile computing," Pervasive Computing, IEEE, vol. 8, no .4, pp. 14-23, 2009.

[15] K. Ha, P. Pillai, W. Richter, Y. Abe, M. Satyanarayanan, "Just-in-time provisioning for cyber foraging," in Proceeding of the 11th annual international conference on Mobile systems, applications, and services. ACM, pp. 153-166, 2013.

[16] Y. Jararweha, L. Tawalbehb, F. Ababneha, A. Khreishahc, F. Dosarib, "Scalable Cloudlet-based Mobile Computing Model," The 11th International Conference on Mobile Systems and Pervasive Computing (MobiSPC-2014). Procedia Computer Science vol. 34, pp. 434-441, 2014.

[17] R. K. Alekberov, F. H. Pashayev, O. R. Alekperov, "Effective Use Method of Cloudlet Resources by Mobile Users," 11th IEEE International Conference on Application of Information and Communication Technologies. Moscow, pp. 401-403, 2017.

[18] M. Jia, W. Liang, Z. Xu, M. Huang, "Cloudlet load balancing in wireless metropolitan area networks," IEEE, INFOCOM, pp. 730-738, 10-14 April 2016.

[19] C. S Hi, V. Lakafosis, M. H. Ammar, E. W. Zegura, "Serendipity: enabling remote computing among intermittently connected mobile devices," in Proc. of the ACM MobiHoc, pp. 145-154. 2012.

[20] R.K. Alekberov, O.R. Alekberov, "Procedure of effective use of cloudlets in wireless metropolitan area network environment," International Journal of Computer Networks \& Communications, vol. 11, no.1, pp. 93-107, 2019.

[21] D. Sarddar, R. Bose, “A Mobile Cloud Computing Architecture with Easy Resource Sharing,” International Journal of Current Engineering and Technology, vol. 4, no. 3, pp. 1249-1254, 2014.

[22] M. Jia, J. Cao, W. Liang, "Optimal Cloudlet Placement and User to Cloudlet Allocation in Wireless Metropolitan Area Networks," IEEE Transactions on Cloud Computing, vol. 5, no .4, pp. 725-737, 2017. 
[23] E. Gelenbe, R. Lent, and M. Douratsos, "Choosing a local or remote cloud," Proceedings of 2nd International Symposium on Network Cloud Computing and Applications, pp.25-30, 2012.

[24] T. Verbelen, P. Simoens, F. D. Turck, and B. Dhoedt, "Cloudlets: Bringing the cloud to the mobile user," Proceedings of 3rd workshop on Mobile Cloud Computing and Services, pp. 29-36, ACM, 2012.

[25] F. Liu, P. Shu, H. Jin, L. Ding, J. Yu, D. Niu, B. Li, "Gearing resource-poor mobile device with powerful clouds: architectures, challenges, and applications," IEEE Wireless Communications. vol. 20, no. 3, pp. 14-22, 2013.

[26] Mikryukov A. A., Khantimirov R. I, "The task of initial resource allocation in cloud computing environments based on the hierarchy analysis method," Applied Informatics. no.8, pp. 184-185, 2015.

[27] Nayyer M. Z., Raza I., Hussain S, "A Survey of Cloudlet-Based Mobile Augmentation Approaches for Resource Optimization," ACM Computing Surveys. vol. 51, no. 5, pp. 1-28, 2018.

[28] Somula R. S., Ra S, A survey on mobile cloud computing: Mobile Computing + Cloud Computing $(\mathrm{MCC}=\mathrm{MC}+\mathrm{CC})$. Scalable Computing: Practice and Experience, vol. 19, no.4, pp. 309-337, 2018.

[29] Ceselli A., Premoli M., Secci S, Mobile Edge Cloud Network Design Optimization. IEEE // ACM Transactions on Networking, vol. 25, no. 3, pp. 1818-1831, 2017.

[30] G. Huerta-Canepa, D. Lee, "A virtual cloud computing provider for mobile devices," International Journal of Advance Research, Ideas and Innovations in Technology, vol. 3, no. 3, pp. 414, 2017.

[31] S. Abolfazli, "Cloud-based Augmentation for Mobile Devices: Motivation, Taxonomies, and Open Challenges," IEEE Commun. Surv. Tutor., vol. 16, pp. 337-368, 2014.

[32] R. K. Alekberov, O. R. Alekberov, "Virtual Machine Selection Algorithm Based on User Requirements in Mobile Cloud Computing Environment," International Journal of Computers \& Technology, vol. 17, no. 2. pp.7 335-7349, 2018.

\section{Authors' Profiles}

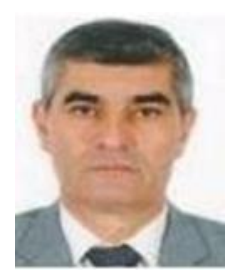

Rashid G. Alakbarov graduated from Automation and Computer Engineering faculty of Azerbaijan Polytechnic University named after C.Ildirim. He received his PhD degree in 2006 from Supreme Attestation Commission under the President of the Republic of Azerbaijan. His primary research interests include various areas in cloud computing, data processing, computer networks, virtual computing, particularly in the area of distributed computing. He is head of department at the Institute of Information Technology as of 2002. Since 2010, he has been leading the development of "AzScienceNet" infrastructure. In 2011, he was appointed a deputy director of the institute by the decision of the Presidium of Azerbaijan National Academy of Sciences. He is the author of 80 scientific papers, including 5 inventions

How to cite this paper: Rashid G. Alakbarov, "Method for Effective Use of Cloudlet Network Resources", International Journal of Computer Network and Information Security(IJCNIS), Vol.12, No.5, pp.46-55, 2020. DOI: 10.5815/ijcnis.2020.05.04 